\title{
Alcohol Intoxication Effects on Simulated Driving: Exploring Alcohol-Dose Effects on Brain Activation Using Functional MRI
}

\author{
Vince D Calhoun*,1,2,3, James J Pekar, ${ }^{4,5}$ and Godfrey D Pearlson ${ }^{1,2,3}$ \\ 'Olin Neuropsychiatry Research Center, Institute of Living, Hartford, CT, USA; ²Department of Psychiatry, Yale University, New Haven, CT, USA; \\ ${ }^{3}$ Department of Psychiatry, Johns Hopkins University, Baltimore, MD, USA; ${ }^{4}$ Department of Radiology, Johns Hopkins University, Baltimore, MD, \\ USA; ${ }^{5}$ FM Kirby Research Center for Functional Brain Imaging, Kennedy Krieger Institute, Baltimore, MD, USA
}

\begin{abstract}
Driving while intoxicated is a major public health problem. We investigated impaired driving using a simulated driving skill game that presents an 'in-car' view of a road and a readout of speed. We explored brain activation and behavioral alterations from baseline at two blood alcohol concentrations (BACs). Participants received single-blind individualized doses of beverage alcohol designed to produce blood alcohol content (BAC) of 0.04 and 0.08 or placebo. Scanning occurred on a 1.5 Tesla Philips MRI scanner after training to asymptote performance. Analysis was performed using independent component analysis (ICA) to isolate systematically nonoverlapping 'networks' and their time courses. Imaging results revealed seven separate driving-related brain networks with different time courses. Several significant findings were observed for the imaging data. First, dose-dependent functional magnetic resonance imaging ( $\mathrm{fMRI}$ ) changes were revealed in orbitofrontal (OF) and motor (but not cerebellar) regions; visual and medial frontal regions were unaffected. Second, cerebellar regions were significantly associated with driving behavior in a dose-dependent manner. Finally, a global disruptive effect of alcohol on the ICA time courses was observed with highly significant differences in OF and motor regions. Alcohol thus demonstrated some behavioral effects and unique, disruptive, dose-dependent effects on $\mathrm{MMRI}$ signal within several brain circuits. The FMRI data also suggest that the deficits observed in alcohol intoxication may be modulated primarily through OF/anterior cingulate, motor and cerebellar regions as opposed to attentional areas in frontoparietal cortex.
\end{abstract}

Neuropsychopharmacology (2004) 29, 2097-2107, advance online publication, I 8 August 2004; doi: I0.1038/sj.npp. 1300543

Keywords: alcohol; $\mathrm{fMRl}$; driving; brain; independent component analysis

\section{INTRODUCTION}

Driving while intoxicated (DWI) remains a persistent public health risk in the United States, resulting in over 17000 fatalities and an estimated 250000 injuries related to intoxicated driving in 2002 (National Highway Traffic Safety Administration, 2002). One of the most common experimental tools in intoxicated driving research is the driving simulator, and many studies to date have employed driving simulators to assess the effects of various abused substances and prescribed medications, as well as the effects of normal aging, sleep deprivation, and other adverse

\footnotetext{
* Correspondence: Dr VD Calhoun, Olin Neuropsychiatry Research Center, Institute of Living, 200 Retreat Avenue, Hartford, CT 06106, USA, Tel: + I 860545 7768, E-mail: vince.calhoun@yale.edu Received 9 February 2004; revised 22 June 2004; accepted 29 June 2004

Online publication: 20 July 2004 at http://www.acnp.org/citations/ Npp07200404054/default.pdf
}

conditions on driving and driving related skills (Linnoila and Mattila, 1973; Rimm et al, 1982; Deery and Fildes, 1999; Arnedt et al, 2001; Verster et al, 2002). We have previously demonstrated the validity of a similar, simulated driving environment to evaluate performance measures in sober and alcohol-intoxicated subjects compared directly to real on-road driving (McGinty et al, 2001).

Acute EtOH effects on behavioral and cognitive functions necessary for driving are well documented (see review of Mitchell, 1985). Alcohol depresses neural activity, with immediate effects on multiple cognitive-motor processing domains (Mongrain and Standing, 1989). Even blood alcohol levels within legal limits produce significant, generalized impairments of multiple attentional abilities; larger doses produce major impairments (Neill et al, 1991). Sustained and divided attentional tasks are most impaired by alcohol (Moskowitz and Sharma, 1974), notably information processing, decision-making, and judgment (Hindmarch et al, 1991). Most alcohol performance decrements are relatively modest, rarely exceeding $35-50 \%$ of unintoxicated baseline performance (Mitchell, 1985). 
Alcohol-related impairment is often dose related but neither identical nor linear for all behaviors.

In a previous study, we applied blood oxygen leveldependent functional magnetic resonance imaging (BOLD fMRI) (Ogawa et al, 1990) to simulated driving, using a driving skill game that presents the participant with an incar' view of a road and a readout of speed (Calhoun $e t$ al, 2002). We analyzed the resulting BOLD data using independent component analysis (ICA), to explore the dynamics of brain networks subserving 'driving.' In the present study, we used these methods to investigate how these networks are affected by alcohol intoxication.

BOLD fMRI yields signals sensitized to hemodynamic sequelae of brain activity, and these time-dependent data are typically reduced, using knowledge of paradigm timing, to graphical depictions of where in the brain the MRI time course resembled the paradigm time course(s) (convolved with a hemodynamic impulse response). This prevalent general linear model (GLM) method (Worsley and Friston, 1995) is a massively univariate inferential approach, as the same hypothesis ('the brain time course resembles the paradigm') is tested at every location (voxel) in the brain.

As the GLM cannot detect brain activity with time courses not known in advance, a variety of multivariate exploratory data analysis (EDA) approaches have been used to reveal patterns in fMRI data. Among the EDA approaches applied to fMRI data, we and others (McKeown et al, 1998; Biswal and Ulmer, 1999; Calhoun et al, 2001b) have shown that spatial ICA is especially useful at revealing brain activity not anticipated by the investigator and at separating brain activity into 'networks' of brain regions with synchronous BOLD fMRI signals. Despite its exploratory nature, the primary assumption behind spatial ICA, that 'different parts of the brain do different things' (Duann et al, 2002), is consistent with the principle of modularity of brain function, and has proven fruitful for the analysis of fMRI data. Temporal ICA, although useful in certain situations, has not yet been performed in whole brain studies due to the computational requirements. In addition, because ICA requires considerable data for reliable source estimates and there are many more voxels than time courses, spatial ICA is the preferable approach for this study (Calhoun et al, 2001b).

In our previous study, ICA extracted six driving-related networks from time-dependent BOLD fMRI data acquired during simulated driving (Calhoun et al, 2002). This analysis revealed sets of brain regions that were differentially activated by simulated driving. Some regions turned on or off, others exhibited a gradual decay, and yet others turned on transiently when starting or stopping driving. Signal in the anterior cingulate cortex, an area often associated with error monitoring and inhibition, decreased exponentially with a rate proportional to driving speed, while decreases in frontoparietal (FP) regions, implicated in vigilance, correlated with speed. Increases in cerebellar and occipital areas, presumably related to complex visuomotor integration, were activated during driving but not associated with driving speed (Calhoun et al, 2002).

Importantly, the activity represented by several of these components (anterior cingulate and FP) was not found when we analyzed our data using the GLM or in a similar fMRI of 'driving' study using only GLM analysis (Walter et al, 2001). This suggests that ICA may be especially useful for analysis of fMRI data acquired during such rich naturalistic behavior, as opposed to the precisely controlled experimental paradigms typical of the fMRI literature where GLM analysis is so prevalent.

In the present study, to investigate how the neural substrates of 'driving' are affected by alcohol intoxication, we applied a novel ICA approach, dubbed 'fixed average spatial' ICA, or FAS-ICA, to data from research participants scanned while driving sober, and under two target concentrations of alcohol. In this approach, ICA was first used to extract driving-related brain networks from sober driving data, and changes in brain activation during DWI were then calculated in these spatial regions.

We had several hypotheses for these data. First, we expected a dose-dependent decrease in behavioral results. Second, we expected to replicate our previous findings with regard to the driving-related components. Third, we expected a global dose-related decrease in fMRI signal (as we found in a previous analysis of a different task; Calhoun et al, 2004). Fourth, we expected to find component-specific EtOH-related disruptions in the temporal dynamics revealed by ICA, specifically in frontal regions and cerebellum. We also expected the same regions to be involved in EtOH-related interactions with the behavioral results.

\section{EXPERIMENTS AND METHODS}

\section{Subjects}

Study participants were nine screened, healthy, righthanded men $(N=7)$ and women, aged $24.2 \pm 5.8$ years. Potential participants were screened with a complete physical and neurological examination, a urine toxicology screen, and the SCAN interview (Janca et al, 1994) to eliminate participants with Axis I psychiatric disorders or history of substance abuse. All participants were nonsmokers, had good visual acuity without correction, valid driver's licenses, good driving records assessed by selfreport, and were light or moderate $(<2$ alcohol consumption sessions/week and $<3$ drinks/session) users of alcohol. Participants were trained to asymptote performance on the simulated driving paradigm. They were requested to eat only a light meal (avoiding fatty foods) the morning of each study (to avoid interfering with alcohol absorption) and to avoid consuming alcohol $24 \mathrm{~h}$ prior to each study day.

An outline of the study design is presented in Figure 1. Participants were scanned in two sessions on two different days. Sessions were run at the same time of day (midmorning). For the first scan session, participants received a placebo after which they were removed from the scanner and received a dose of beverage alcohol individualized to participant body weight, age, and sex, calculated using a published algorithm (Kapur, 1989), and designed to produce a blood alcohol concentrations (BACs) of 0.04 or $0.08 \%$ (the dose was counterbalanced between days 1 and 2). The alcohol beverage was administered orally in singleblind manner as $190 \mathrm{pf}(95 \%(\mathrm{v} / \mathrm{v}))$ ethanol diluted in chilled fruit juice to a constant volume, consumed over $10 \mathrm{~min}$. Placebo drinks were of the same total volume, including $15 \mathrm{~cm}^{3}$ of alcohol floating on the surface. All beverages were served in identical glass containers, wrapped 

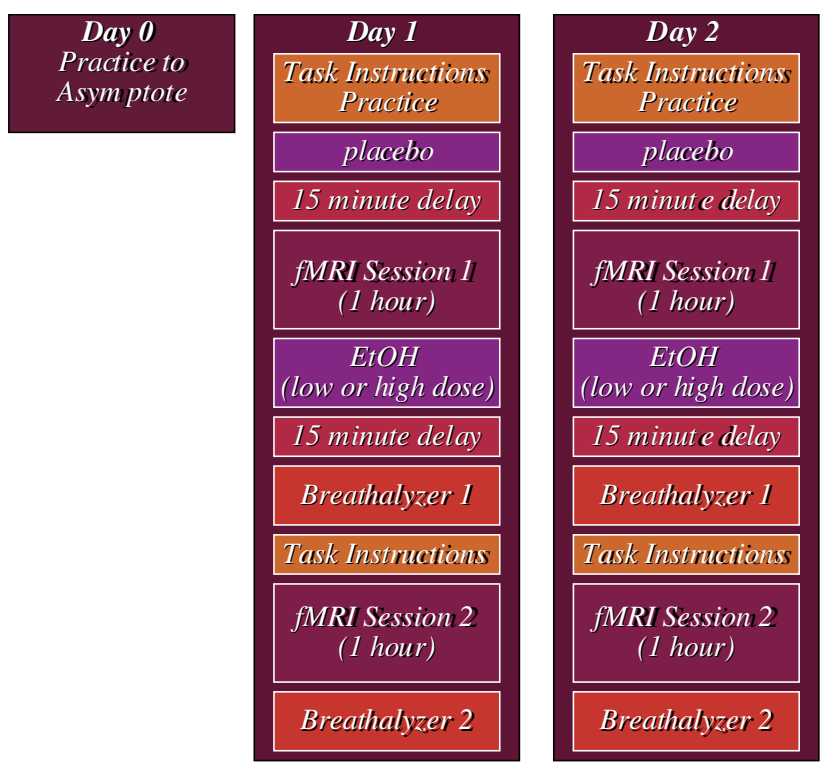

Figure I Study design. Outline of study design consisting of 2 days with two scan sessions on each day. Boxes are not proportional to the amount of time spent on each task.

in an alcohol-soaked cloth to help disguise the contents (Hammersley et al, 1992). BACs were determined immediately before and after the scan session, using a hand-held breath meter (Intoximeters Inc.), and subjects were blind to BACs.

Participants began their test sessions 20 min postbeverage. Each was run in two separate sessions, on two separate days, randomly, one at each alcohol blood level, always preceded by a placebo run. Subjects self-rated level of subjective intoxication on a verbal analog ( $0-5$ point) scale. A licensed physician oversaw dosing and administration. Following completion of each scan session, participants were compensated for their time plus an additional sum based on their driving performance. This latter amount was for obeying road rules (eg keeping to posted speed limits). The Johns Hopkins Medicine Institutional Review Board approved the protocol, and all participants provided written informed consent.

\section{Experimental Design}

Methods are those described previously in Calhoun et al (2002). We obtained fMRI scans of subjects as they twice performed a 10-min task consisting of 1-min epochs of: (a) an asterisk fixation task, (b) active simulated driving, and (c) watching a simulated driving scene (while randomly moving fingers over the controller). Epochs (b) and (c) were switched in the second run and the order was counterbalanced across subjects. During the driving epoch, participants were performing simulated driving using a modified game pad controller with buttons for left, right, acceleration, and braking. The paradigm time line is illustrated in Figure 2. Subjects were instructed to remain within a predetermined speed range and were compensated additionally if they successfully achieved this goal.

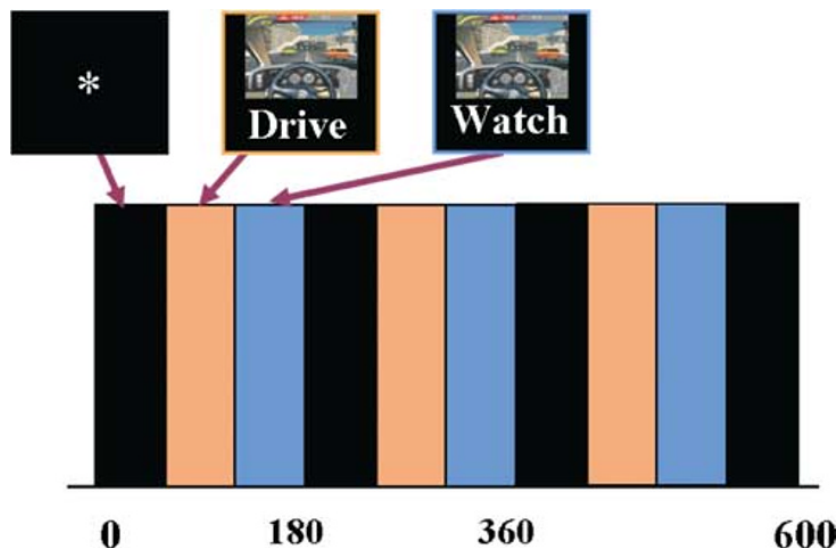

Figure 2 fMRI-simulated driving paradigm. The paradigm consisted of 10, I-minute epochs of (a) a fixation target, (b) driving the simulator, and (c) watching a simulation while randomly moving fingers over the controller. The paradigm was presented twice changing the order of the (b) and (c) epochs and counterbalancing the first order across subjects.

The simulator used was a commercially available driving game, Need for Speed II $^{\mathrm{TM}}$ (Electronic Arts, 1998). The controller was shielded in copper foil and connected to a computer outside the scanner room though a waveguide in the wall. All ferromagnetic components were removed and replaced by plastic. An LCD projector outside the scanner room and behind the scanner projected through another waveguide to a translucent screen, which the subjects saw via a mirror, attached to the head coil of the fMRI scanner. The screen subtended approximately $25^{\circ}$ of visual field. The watching epoch was the same for all subjects (a playback of a previously recorded driving session). For the driving epoch, subjects started at the same point on the track with identical conditions (eg car type, track, traffic conditions). They were instructed to stay in the right lane, except in order to pass, to avoid collisions, to stay within a speed range of 100-140 (the units were not specified), and to drive normally.

\section{Rating of Driving Performance}

As NFS-II allows driving sessions to be played back, two independent raters, blind to subject identity and experimental condition, separately scored each driving session on eight parameters. Inter- and intrarater reliability using intraclass correlation coefficients for those ratings exceeded 0.85 on five randomly chosen subject sessions rated twice, blind to subject identity. Parameters assessed included indices of speeding, weaving, collisions, etc as shown in Figure 3. Ratings of the two independently rated scores were averaged for each run.

\section{FMRI Data Acquisition}

Data were acquired at the FM Kirby Research Center for Functional Brain Imaging at Kennedy Krieger Institute on a Philips NT 1.5 Tesla scanner. A sagittal localizer scan was performed first, followed by a T1-weighted anatomic scan $(\mathrm{TR}=500 \mathrm{~ms}, \mathrm{TE}=30 \mathrm{~ms}$, field of view $=24 \mathrm{~cm}$, matrix $=256 \times 256$, slice thickness $=5 \mathrm{~mm}$, gap $=0.5 \mathrm{~mm}$ ) 


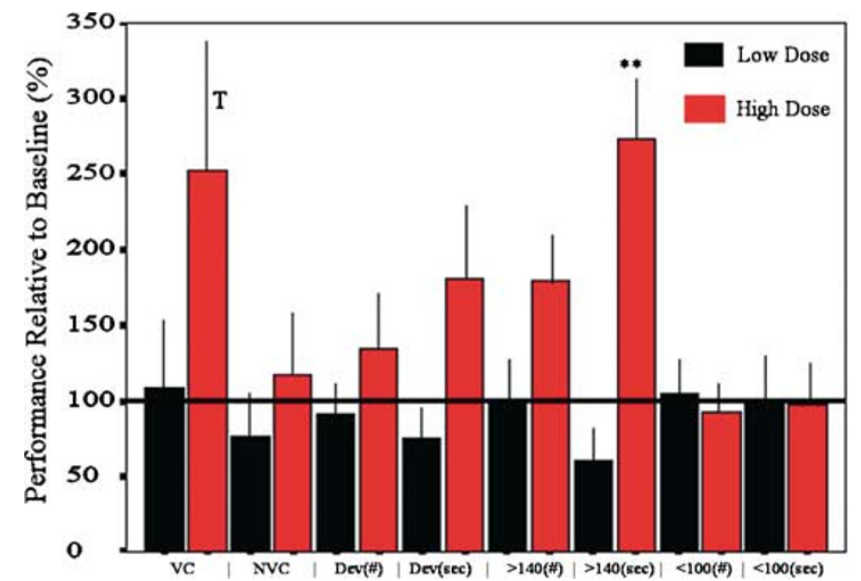

Figure 3 Behavior results from the driving data. Driving performance at low- and high-dose EtOH (black and red bars) compared to (mean) sober performance (solid black bar) rated on seven different measures. Measured included vehicle collisions (VC), near vehicle collisions (NVC), lane deviations $(\operatorname{DEV}(\#))$, length of lane deviations $(\operatorname{Dev}(s))$, number of times over speed limit $(>\mid 40(\#))$, time over speed limit $(>\mid 40(s))$, number of times below speed limit $(<100(\#))$, and time below speed limit $(<100(s))$

consisting of 18 slices through the entire brain including most of the cerebellum. Next, we acquired the functional scans consisting of an echo-planar scan $(\mathrm{TR}=1 \mathrm{~s}$, $\mathrm{TE}=39 \mathrm{~ms}$, field of view $=24 \mathrm{~cm}$, matrix $=64 \times 64$, slice thickness $=5 \mathrm{~mm}$, gap $=0.5 \mathrm{~mm}$ ) consisting of 18 slices obtained consistently over a 10 -min period per run for a total of 600 scans. In all, 10 'dummy' scans were performed at the beginning to allow for longitudinal equilibrium, after which the simulated driving paradigm was begun.

\section{FMRI Analysis Overview}

We were interested in an examination of the intoxicationinduced changes in the temporal dynamics of drivingrelated networks (see Calhoun et al, 2002). To do this, we first perform an ICA analysis on the $9 \times 2$ sober-condition data sets to generate a set of maximally independent images. We then computed ICA time courses for all four fMRI sessions and nine participants using these fixed component images, appropriately called a FAS ICA approach. For comparison, we also performed a typical GLM analysis assuming fixed responses during each condition and convolving with the default hemodynamic response in the SPM2 software package.

\section{FMRI Data Preprocessing}

The images were first corrected for timing differences between the slices using windowed Fourier interpolation to minimize the dependence upon which reference slice is used (van de Moortele et al, 1997; Calhoun et al, 2000). Next, the data were imported into the Statistical Parametric Mapping software package, SPM99 (Worsley and Friston, 1995). Data were motion corrected, spatially smoothed with an $8 \times 8 \times 11 \mathrm{~mm}^{3}$ Gaussian kernel, and spatially normalized into the standard space of Talairach and Tournoux (1988). The data were slightly subsampled to $3 \times 3 \times 4 \mathrm{~mm}^{3}$, resulting in $53 \times 63 \times 28$ voxels. For display, slices two to 26 were presented.

\section{Independent Component Analysis}

For the ICA estimation, we used our group ICA approach (Calhoun et al, 2001a). To review briefly, computing a separate ICA analysis of each participant does not lend itself well to group analysis since it is unclear as to which components it is to be compared with. In this approach, a single ICA analysis is performed on a group of participants, followed by a back reconstruction of single-subject time courses and spatial maps from the raw data. Two data compression stages are used, first at the level of the individual participant and second on the subject-wise concatenated data. This subject-wise concatenation approach has been shown to be the preferable approach to group ICA analysis (Calhoun et al, 2001a; Schmithorst and Holland, 2004). For group ICA, data from each subject are reduced from 600 to 30 time points using principal component analysis (PCA) (representing greater than 99\% of the variance in the data). Data from all subjects are then concatenated, and this $30 \times$ (number of data sets) aggregate data set reduced to 25 dimensions using PCA, followed by independent component estimation using a neural network algorithm that attempts to minimize the mutual information of the network outputs (Bell and Sejnowski, 1995). Time courses and spatial maps are then reconstructed for each subject. Our methods are available in a Group ICA of FMRI Toolbox (GIFT) implemented in Matlab (http:// icatb.sourceforge.com).

We then calculate the average sober-condition ICA maps for each of the 25 components and, using these 25 images, we generate single-subject component time courses for all data sets using spatial multiple regression of the component images onto the fMRI data for each time point (that is, we flatten each fMRI volume/time point into a row vector and compute the regressor of the spatial component with the fMRI volume and a spatial intercept regressor). We are thus computing a multiple regression of all components (and all voxels) onto the data at once as a way to generate ICA time courses from component images. This approach is distinct from a regions-of-interest approach such as what might be utilized in a typical GLM analysis. Rather, given a set of (fixed) independent component images, we essentially generate their companion time courses.

The resulting single-subject time-course amplitudes were then calibrated (scaled) using the raw data so that they reflected percent fMRI signal strength (Calhoun et al, 2001a) and can be compared between the groups. Finally, we selected the components that were highly correlated with the paradigm for further analysis.

\section{Analyses of Temporal Dynamics}

A statistical examination of the ICA time courses (for each component we had nine participants and four scans session for a total of 36 time courses/component) was then performed. The first analysis involved parameterizing the ICA time courses and testing for dose-related changes in these parameters (regardless of behavior). A second analysis examined the relationship of the ICA time-course 
parameters with two behavioral measures. The third analysis involved computing a measure of EtOH doserelated disruption in the ICA time courses.

Analysis 1: $f M R I / E t O H$. In order to parameterize the ICA time courses, we grouped them into three main patterns (see Figure 8 for a visual summary of the patterns). First, primary visual (yellow) and higher-order visual (white) areas were most active during driving and less active during watching (as compared to fixation); second, cerebellar (orange with turquoise border), motor (red), and FP (blue) areas were only changed during driving; finally, anterior cingulate medial frontal and orbitofrontal (OF) areas (pink) demonstrated exponential decrements during driving and rebounded during fixation. We then parameterized these components according to their patterns as follows. The first pattern was represented by the average of the middle $40 \mathrm{~s}$ (to avoid transition points) of the drive condition subtracted from the average of the middle $40 \mathrm{~s}$ of the fixation condition (D_40-F_40) and also the drive condition subtracted from the watch condition (D_40-W_40). The second pattern was represented by D_40-FW_40 (the average of the middle $40 \mathrm{~s}$ of both the fixation and watch conditions). The third pattern was represented by an exponential rate parameter determined by fitting the driving epoch of the time courses to a single exponential using the Gauss-Newton method implemented in Matlab.

Analysis 2: behavior/fMRI/EtOH. We also examined the relationship of these parameterized ICA time courses to the significant behavioral measures. In addition, because we previously found a significant correlation of driving speed to the rate of exponential decrease in the OF component, we performed a similar analysis to examine correlations with drug dose.

Analysis 3: disruption score/EtOH. For the analysis of the entire ICA time course, we computed within-day correlation scores (ie sober condition was correlated with the same-day low- or high-dose condition) for each component as a general measure of the amount of 'disruption' in the time courses induced by the alcohol challenge. A test for changes in these disruption scores for the two alcohol doses was then performed.

\section{GLM Analysis}

The GLM analysis included the following steps: (1) creation of 'box-car' regressors for the driving and watching conditions, (2) convolution of this idealized regressor with a canonical hemodynamic response function consisting of the sum of two gamma functions, (3) temporal regression of these regressors (plus a temporal intercept regressor) onto the fMRI data for each voxel to generate single-subject parameters, and (4) second-level 'random-effects' analysis using voxel-wise $t$-tests to examine which locations exhibit the predicted time courses (Holmes and Friston, 1998).

\section{RESULTS}

We explored the effect of alcohol (EtOH) at two BACs on simulated driving performance. At the lower BAC (mean $0.041 \pm 0.016$ ), on the five-point analog scale (where five indicated maximal intoxication), participants indicated subjective intoxication of mean $1.0 \pm 0.7$, and at the higher BAC (mean 0.096 \pm 0.040 ), participants self-rated intoxication of mean $3.1 \pm 0.8$. The difference on the subjective intoxication scores was highly significant $(p<0.000001)$.

\section{Behavioral Results}

Driving performance was rated on eight different measures: vehicle collisions, near vehicle collisions, number of lane deviations, duration of lane deviations, number of instances over maximum speed limit, total time over maximum speed limit, number of instances below minimum speed limit, and total time below minimum speed limit. Driving performance outcome measures thus indicated deficits in lateral and longitudinal vehicle control. Compared to sober baseline, at the lower BAC, performance slightly improved and participants reduced average speed. At the higher BAC, subjects drove more at higher speeds $(p<0.008$, corrected) and there was a trend toward increased collisions with other cars $(p<0.15$, corrected). All $p$-values are corrected for multiple comparisons unless otherwise noted. Figure 3 depicts driving performance results for eight measures in the drug condition relative to the sober condition.

\section{GLM Results}

For the fMRI motion correction, the translation corrections were less than half a voxel for any participant. Pitch, roll, and yaw rotations were generally within $2.0^{\circ}$ or less. There were no visually apparent differences between the average motion parameters for the sober and alcohol intoxication runs. Changes were also not significant as assessed by a $t$ test on the sum of the squares of the parameters over time. The GLM fMRI analysis results for the sober condition are presented in Figure 4. Relative increases are in red and decreases in blue. Networks correlated with the drive regressor are depicted on the left and networks correlated with the watch regressor are on the right. We found, as reported by others, that motor and cerebellar networks were significantly different in the contrast between drive and watch (Walter et al, 2001). We also performed a contrast between the intoxicated condition (high or low dose) and the sober condition in order to examine whether differences in spatial location or amplitude were evident. Specifically, we computed voxel-wise contrasts (in both directions) for '(drive sober-fixation sober)-(drive high dose-fixation high dose)', '(drive sober-fixation sober)-(drive low dose-fixation low dose)' and similarly for the watch regressor. One-sample $t$-tests on the resulting contrasts did not reveal significant differences between the two conditions.

\section{ICA Image Results}

ICA imaging results from the FAS analysis are summarized in Figure 5, with different colors coding for each 

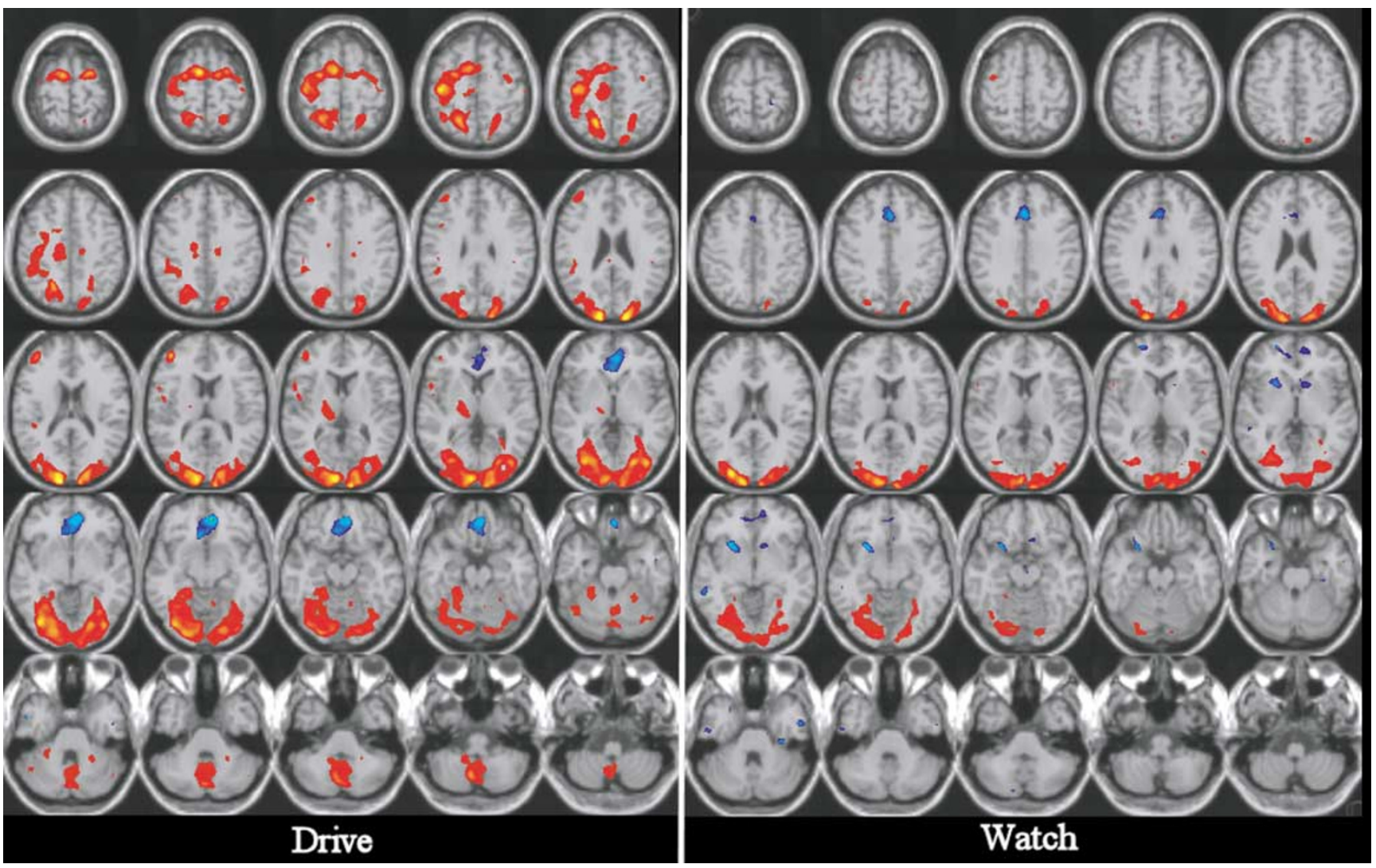

Figure 4 Results from the GLM-based fMRI analysis. Regression increases (red) and decreases (blue) are depicted for the driving condition and the watch condition (same colors but surrounded with black border). There were considerably more motor increases and OF decreases during the driving condition. The extent of activation in most regions is greater for the driving condition.

component. Owing to the low degrees of freedom (8), we used a corrected threshold of $p<0.005(t=4.5)$, which controls for the false discovery rate (Genovese et al, 2002). The average time courses for each scan session are presented in the middle and right panels of Figure 5, respectively. For visualization, the three epoch cycles are averaged together and are presented as 'fixation', 'drive', and 'watch'. The 25 time courses were sorted according to their correlation with the driving paradigm, and then visually inspected for task-related or transiently task-related activity (based on their periodicity with the paradigm). Of the 25 components, seven demonstrated such a relationship. This is consistent with previous results (Calhoun et al, 2002), with the exception that the primary/supplemental motor and cerebellar networks were divided into two separate components (albeit with similar temporal patterns on average).

The left panel of Figure 5 displays the thresholded average components-of-interest (ie 'weighted regions-of-interest (networks)') determined from the sober-condition data. Cerebellar networks are depicted in the same color as the motor and supplementary motor regions, except with a turquoise border surrounding the region. The middle panel displays the average calibrated time courses (computed via spatial regression of the component maps onto the raw data for each subject) from the sober- and low-dose condition, with color use as in the spatial component maps (the drug condition is indicated with the color gray). The right panel display is the same for the fMRI session consisting of the sober condition and corresponding (same day) high-dose condition (also indicated with the color gray). Selected Talairach coordinates of the volume and maxima of each anatomic region within the maps are presented in Table 1.

Analysis 1: $f M R I / E t O H$. For all participants, the fit to a single exponential was quite good (average $p<0.00005$ ). The parameterization by dose analysis was only significant for the OF rate ${ }^{-1}$ parameter. Figure 6 is a plot of the OF component time courses fitted rate $^{-1}$ parameter as a function of scan session. The rate ${ }^{-1}$ is larger at both alcohol doses than in the sober condition. There is a significant $(p<0.005$, corrected) change between the sober condition and the high-dose condition (the change in the low-dose condition is a trend in the same direction).

Analysis 2: behavior/fMRI/EtOH. A direct comparison of significant behavioral measures (vehicular collisions and speed over 140), EtOH dose, and fMRI signal was performed by computing the dose-related correlation of the behavior results with these parameterized results. All $p$-values were greater than 0.2, except for two results: the cerebellar component (D_40-FW_40) exhibited a highly significant dose-related affect with driving greater than $140(p<0.001$, corrected) and the FP-cingulate component (D_40-FW_40) showed a weak dose-related correlation with vehicular collisions $(p<0.15$, corrected $)$. 

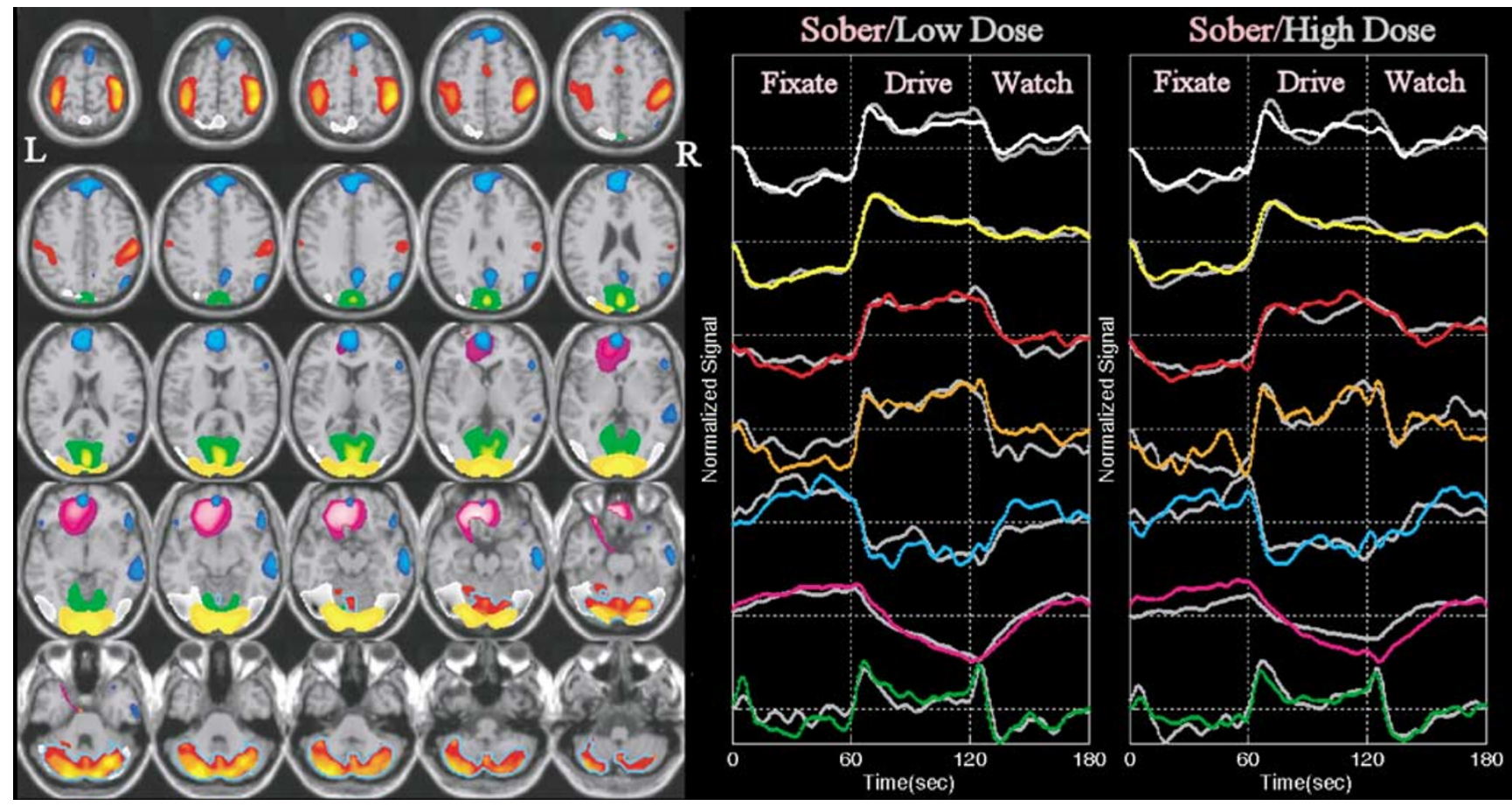

Figure 5 Results from the FAS analysis. Random effects group fMRI maps are thresholded at $p<0.005$. A total of seven components are presented. A 'green' component extends on both sides of the parieto-occipital sulcus including portions of cuneus, precuneus, and the lingual gyrus. A 'yellow' component contains mostly occipital areas. A 'white' component contains bilateral visual association and parietal areas; and a component consisting of motor areas is depicted in red. Cerebellar areas are also depicted in red (but with a turquoise border). OF and anterior cingulate areas identified are depicted in pink. Finally, a component including medial frontal, parietal, and posterior cingulate regions is depicted in blue. Group averaged time courses (right) for the fixate drive watch order are also depicted with similar colors for the sober vs low-dose and sober vs high-dose conditions (drug conditions are colored gray). The three repeated epochs are averaged and presented as fixation, drive, and watch.

Analysis 3: disruption score/EtOH. The change in the fMRI disruption score with respect to dose is shown in Figure 7 (coloring is identical to that used in Figure 5). All components were found to be temporally less correlated for the high-dose experiment than for the low-dose experiment. Significant differences were found for the OF component and the primary motor/SMA component $(p<0.001)$. The cerebellar component was the least affected by alcohol dose for the correlation analysis.

\section{DISCUSSION}

We studied the impact of two doses of EtOH upon behavioral and fMRI data during performance of a simulated driving task (NFS-II). From NFS-II, we derived eight highly reliable quantitative driving performance measures. Behavioral results of the present study revealed mild opposite effects of low- and high-dose $\mathrm{EtOH}$ on driving behavior (consistent with a previous study; McGinty et al, 2001), which we interpret as successful compensation for awareness of mild alcohol-induced intoxication (although additional data are needed to draw stronger conclusions from this trend).

ICA revealed a set of driving-related networks. Consistent with previous results (on different participants), several time courses were modulated by the driving task (Calhoun et al, 2002). The components identified by our analysis lend themselves naturally to interpretation in terms of well- known neurophysiological networks as discussed in (Calhoun et al, 2002). In Figure 8, the seven components are divided into four patterns with $\mathrm{EtOH}$ and speed-related effects (Calhoun et al, 2002) indicated. Hypothesized functions of these networks are: (1) vigilance, (2) error monitoring and inhibition, (3) motor, (4) higher-order motor (cerebellar), (5) visual, (6) higher-order visual, and (7) visual monitoring. We delineated the networks affected by driving speed in the previous study, as well as the networks affected by alcohol dose in the current study. We have discussed the involvement of these circuits in the context of simulated driving in detail previously, and the reader is referred to this work for further discussion (Groeger, 2000; Calhoun et al, 2002).

Consistent with our hypotheses significant alcohol-related changes were found in $\mathrm{OF} /$ anterior cingulate cortex and primary motor cortex/SMA. The medial OF cortical involvement in alcohol dose is consistent with a recent EEG study demonstrating that alcohol consumption impaired error detection in this region using (Ridderinkhof et al, 2002).

Owing to a significant speed-related relationship to both the OF rate parameter and the D_40-FW_40 activity in the OF component in a previous study (Calhoun et al, 2002), we suggested that both the vigilance network and the error monitoring network could be interpreted as a 'switching' influence of the vigilance network upon the error monitoring network. In this context, awareness of driving 
Table I Areas Identified by the FAS Analysis

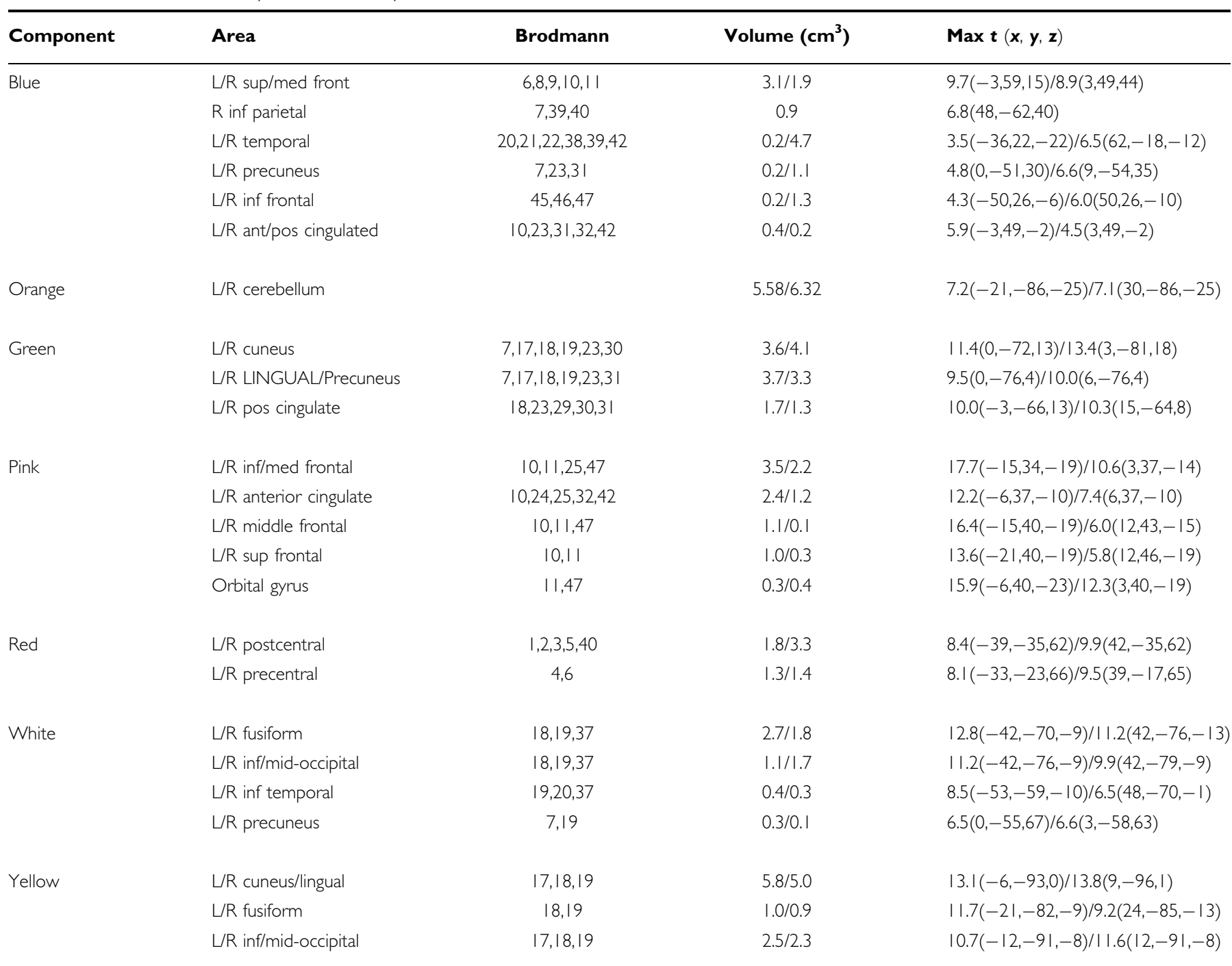

For each component, all the voxels above the threshold were entered into a database to provide anatomic and functional labels for the left $(L)$ and right $(R)$ hemispheres. The volume of activated voxels in each area is provided in cubic centimeters $\left(\mathrm{cm}^{3}\right)$. Within each area, the maximum $t$ value and its coordinate are provided.

(vigilance) is initiated once the driving condition begins (manifesting as an exponential decrease in fMRI signal). Error correction and disinhibition are revealed as a gradual decline of this component at a rate determined, in part, by the vigilance network. When subjects drive faster, the vigilance component changes more; thus, the error correction and disinhibition component decreases at a faster rate. In the present study, the speed was not modulated to a large degree (although a small but significant increase in the high-dose condition was observed). However, the $\mathrm{OF}$ network was modulated by EtOH, but the FP network was not. Thus, the current results are consistent with a dissociation of the OF and FP networks (the latter network is modulated by alcohol, whereas the former network is not) manifesting as a problem in the OF network primarily (whereas the FP network does not appear to be affected).

Contrary to hypotheses, the cerebellum was not significantly affected on the previous measure. The cerebellum did, however, exhibit a highly significant dose-related correlation with the number of times the speed limit was exceeded (specifically the fMRI amplitude was more correlated with speed limit excesses for the high-dose session than for the low-dose session). This may be due to less efficient processing of the complex motor coordination required at higher speeds. At BACs approaching $0.10 \%$, there is a significant increase in collisions or near collisions (Voas and Tippetts, 1999; Weiler et al, 2000). The doserelated cerebellar involvement is also consistent with previous studies implicating a detrimental effect of alcohol upon complex motor control (Peterson et al, 1990) and alcohol-associated decreases in cerebellar blood flow (Volkow et al, 1988). As visual and attentional fields narrow, inebriated drivers are less responsive to peripheral events and often exhibit depressed perceptual and motor functioning (Mascord et al, 1995). We did not find significant differences in the FP region, contrary to the 


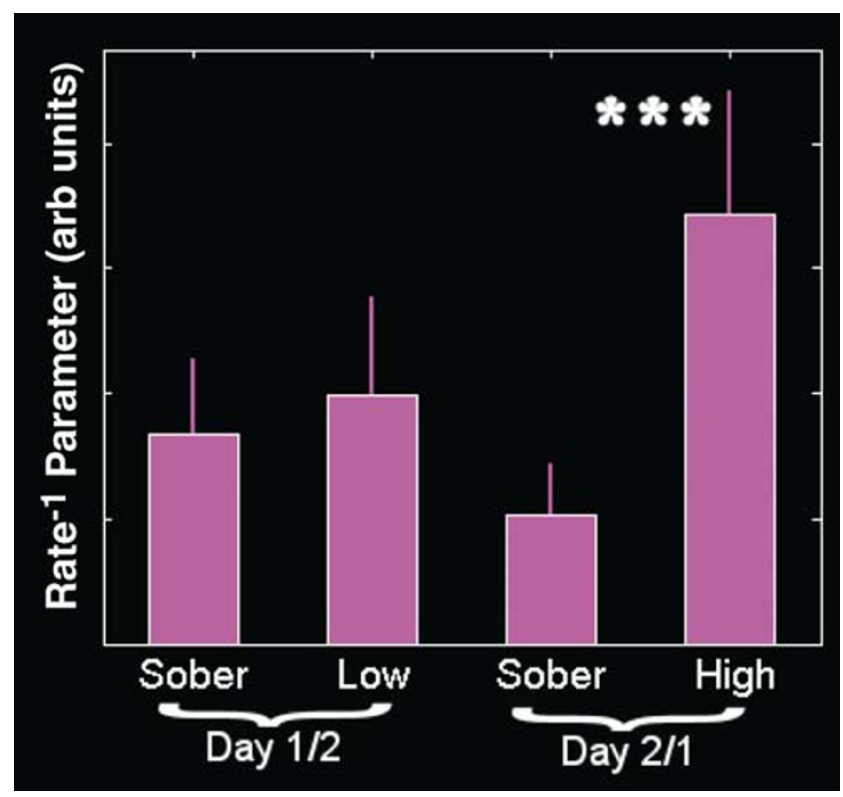

Figure 6 Exponential fit to orbitofrontal component time course. Mean rate-parameter fit to the exponentially decreasing orbitofrontal component time course as a function of $\mathrm{EtOH}$ dose. The rate of decrease was significantly $(p<0.005)$ less in the high-dose condition than the sober condition (with a trend in the same direction for the low-dose condition).

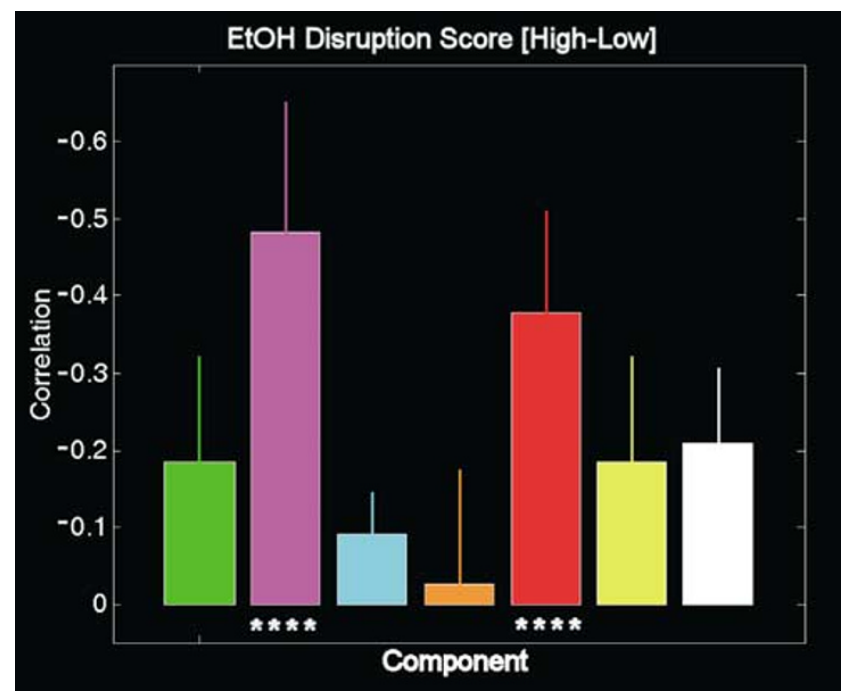

Figure 7 Differences in disruption scores for the ICA time courses. Within-day correlations were computed between the sober condition and the drug condition on the same day as a measure of the amount of disruption induced by the $\mathrm{EtOH}$. The differences in these correlations are presented for each component with color corresponding to Figure 5. The high-dose condition was in all cases less correlated with its sober counterpart than was the low-dose condition (all values are negative). Significant $(p<0.00 \mathrm{I})$ differences were observed for the pink (OF/anterior cingulate) and red (motor) components only.

hypotheses. The lack of significant findings in these regions, coupled with findings for the other regions discussed, suggests that decreased motor functioning during intoxication may be due less to attentional differences than to the impact of EtOH on cerebellar/motor and OF regions.

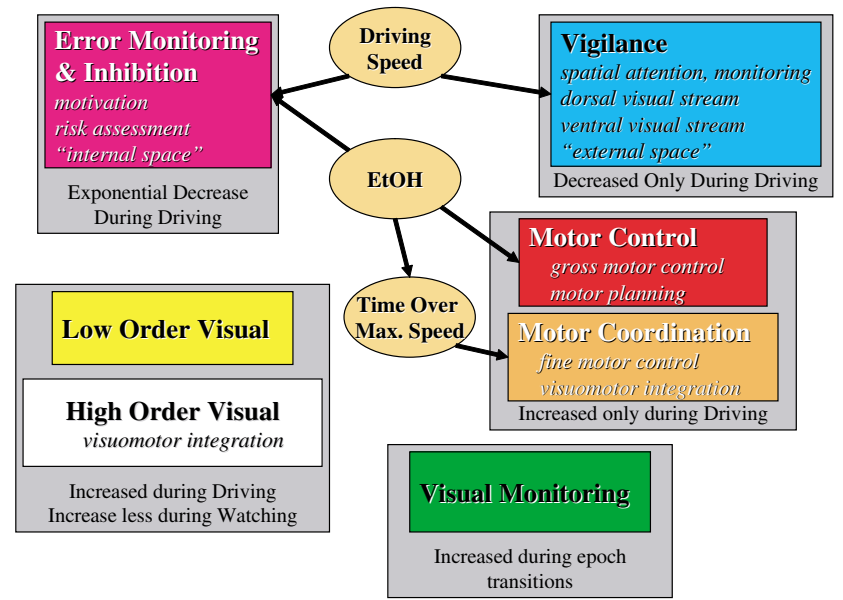

Figure 8 Interpretation of imaging results. Extension of the results of our previous study examining speed-related changes (Calhoun et al, 2002). Colors correspond to those used in Figure 5. Components are grouped according to the averaged pattern demonstrated by their time courses. The speed modulated components (previously found) as well as the EtOH component revealed in the present study are indicated with arrows.

Results from the GLM analysis were consistent with the ICA results (and with a previous study; Walter et al, 2001), in that fewer regions were activated during passive watching than during simulated driving. Compared with ICA, similar (but fewer) regions were revealed, and the temporal regression analysis is unable to reveal separate networks (even when ICA time courses are used as regressors) because of the high degree of temporal correlation between the time courses. In addition, no significant alcohol-related changes were detected with the regression analysis, presumably because the regression analysis was unable to capture the complexity of the temporal behavior during simulated driving. As only nine participants were included in the study a fixed-effects analysis was also performed, with similar results. Although it would be informative to try to separate finer temporal correlates within the ICA time courses (such as the time of a crash), we do not have exact timing for individual events in the simulator used and, thus, we limited our exploration to epoch-based averages. Future studies will use event-related fMRI designs to explore these issues.

Alcohol is known to have vasoactive properties, potentially confounding BOLD fMRI, which relies upon hemodynamic changes. Global changes (more specifically decreases) in fMRI signal changes are likely confounded by such vasoactive changes. A previous fMRI study reported that alcohol resulted in a significant activation decrease in visual areas, slightly more on the right side (Levin et al, 1998). We did not find significant global alcohol-related changes for the driving paradigm (see, for example, the yellow time courses in Figure 5). However, we did find that a higher EtOH dose decreased the within-day correlation for all components (significantly so for two components), suggesting that alcohol has a disruptive effect upon brain activation. This finding differs from a prior fMRI study (collected at the same time as the simulated driving data), in which we examined alcohol effects on a visual perception task and found a general decrease in contrast-to-noise ratio 
in some regions as well as dose-related changes (both increases and decreases) in other regions (Calhoun et al, 2004), suggesting that the global EtOH effect may be a function of the task used.

In our previous study, the cerebellar and motor networks were part of a single component (Calhoun et al, 2002). The present study distinguished these as separate components, although their time courses were quite similar (on average). The present study had two sober driving conditions for each participant, and the fact that we can discriminate between cerebellar and motor networks may then reflect a sensitivity issue given that we have twice as much data. In both studies, 25 components were estimated, but we also verified that the results were stable by changing the number of components estimated from 20 to 35 , and this did not change the fact that cerebellar and motor networks were divided into two components.

A limitation of the FAS ICA approach is that the identified networks are potentially biased towards the sober condition because they were derived from the sober condition data. In order to rule out this potential bias problem, we performed two additional analyses: (1) the data from the low and high $\mathrm{EtOH}$ dose were analyzed with group ICA and (2) the entire experiment was analyzed with group ICA. In each case, the results showed similar findings to the FAS analysis using the sober condition as a reference. An additional limitation of the present study is the relative small number of participants. Our findings, although highly significant, merit subsequent studies with a larger sample size.

The fact that at the high $\mathrm{BAC}$ participants tended to drive faster was recognized as a potential confound, given our previous finding of a correlation between the $\mathrm{OF}$ region and driving speed. However, we do not think faster driving between sober and alcohol conditions explained the observed changes for the following reasons: (1) only the $\mathrm{OF}$ (and not the FP) region is associated with alcohol dose, (2) the speed range was more restricted than in our earlier study (all had same speed range in the present study), and (3) although there was a trend towards decreased speed at the low dose, the OF region showed a trend toward increased rate (the opposite of the effect we demonstrated speed would have in our earlier paper).

In conclusion, we have replicated earlier fMRI results revealing different activation dynamics for multiple regions during a simulated driving task. We have studied the impact of two doses of $\mathrm{EtOH}$ upon these dynamics using a proposed fixed average spatial analysis of these networks. In addition, we have implicated OF/anterior cingulate and primary motor networks as being modulated by $\mathrm{EtOH}$ in a dose-related manner and the cerebellar network as associated with speeding in a dose-related manner. The fMRI data suggest that the attentional deficits observed in $\mathrm{EtOH}$ intoxication may be modulated through the $\mathrm{OF}$ and cerebellar regions as opposed to attentional areas in FP regions.

\section{ACKNOWLEDGEMENTS}

We thank Dave Altschul, Vince McGinty, Dave Scott, Mohammed Shaikh, Heather Strasser, Regina Shih, and Todd Watson for help with data collection. Data were acquired at the FM Kirby Research Center for Functional Brain Imaging, Kennedy Krieger Institute. This work was supported by the National Institutes of Health under Grant 1 R01 EB 000840-01 (to VC), by an Outpatient Clinical Research Centers Grant M01-RR00052 (to GP), and by P41 RR 15241.

\section{REFERENCES}

Arnedt JT, Wilde GJ, Munt PW, MacLean AW (2001). How do prolonged wakefulness and alcohol compare in the decrements they produce on a simulated driving task? Accid Anal Prev 33: 337-344.

Bell AJ, Sejnowski TJ (1995). An information maximisation approach to blind separation and blind deconvolution. Neural Comput 7: 1129-1159.

Biswal BB, Ulmer JL (1999). Blind source separation of multiple signal sources of fMRI data sets using independent component analysis. J Comput Assist Tomogr 23: 265-271.

Calhoun VD, Adali T, Kraut M, Pearlson GD (2000). A weightedleast squares algorithm for estimation and visualization of relative latencies in event-related functional MRI. Magn Res Med 44: 947-954.

Calhoun VD, Adali T, Pearlson GD, Pekar JJ (2001a). A method for making group inferences from functional MRI data using independent component analysis. Hum Brain Mapp 14: 140-151.

Calhoun VD, Adali T, Pearlson GD, Pekar JJ (2001b). Spatial and temporal independent component analysis of functional MRI data containing a pair of task-related waveforms. Hum Brain Mapp 13: 43-53.

Calhoun VD, Altschul D, McGinty V, Shih RA, Scott D, Pearlson GD (2004). Alcohol intoxication effects on visual perception: an fMRI study. Neuroimage 21: 15-26.

Calhoun VD, Pekar JJ, McGinty VB, Adali T, Watson TD, Pearlson GD (2002). Different activation dynamics in multiple neural systems during simulated driving. Hum Brain Mapp 16: 158-167.

Deery HA, Fildes BN (1999). Young novice driver subtypes: relationship to high-risk behavior, traffic accident record, and simulator driving performance. Hum Factors 41: 628-643.

Duann JR, Jung TP, Kuo WJ, Yeh TC, Makeig S, Hsieh JC et al (2002). Single-trial variability in event-related BOLD signals. Neuroimage 15: 823-835.

Electronic Arts I (1998). Need for Speed II.

Genovese CR, Lazar NA, Nichols T (2002). Thresholding of statistical maps in functional neuroimaging using the false discovery rate. Neuroimage 15: 870-878.

Groeger J (2000). Understanding Driving: Applying Cognitive Psychology to a Complex Everyday Task. Psychology Press: New York.

Hammersley R, Finnigan F, Millar K (1992). Alcohol placebos: you can only fool some of the people all of the time. Br J Addict 87: $1477-1480$.

Hindmarch I, Kerr JS, Sherwood N (1991). The effects of alcohol and other drugs on psychomotor performance and cognitive function. Alcohol Alcohol 26: 71-79.

Holmes AP, Friston KJ (1998). Generalizability, random effects, and population inference. Neuroimage 7: S754.

Janca A, Ustun TB, Sartorius N (1994). New versions of World Health Organization instruments for the assessment of mental disorders. Acta Psychiatr Scand 90: 73-83.

Kapur BM (1989). Computer Blood Alcohol Calculator v1.20 ARF Software. Addiction Research Foundation: Toronto, Canada.

Levin JM, Ross MH, Mendelson JH, Kaufman MJ, Lange N, Maas LC et al (1998). Reduction in BOLD fMRI response to primary visual stimulation following alcohol ingestion. Psychiatry Res 82: 135-146. 
Linnoila M, Mattila MJ (1973). Interaction of alcohol and drugs on psychomotor skills as demonstrated by a driving simulator. $\mathrm{Br} \mathrm{J}$ Pharmacol 47: 671P-672P.

Mascord D, Walls J, Starmes G (1995). In: Hartley L (ed). Fatigue and Driving: Driver Impairment, Driver Fatigue, and Driving Simulation. Taylor \& Francis: London. pp 189-205.

McGinty VB, Shih RA, Garrett ES, Calhoun VD (2001). Assessment of intoxicated driving with a simulator: a validation study with on road driving. Proceedings of the Human Centered Trans Sim Conference. Iowa City, IA. p 11.

McKeown MJ, Makeig S, Brown GG, Jung TP, Kindermann SS, Bell AJ et al (1998). Analysis of fMRI data by blind separation into Independent spatial components. Hum Brain Mapp 6: 160-188.

Mitchell MC (1985). Alcohol-induced impairment of central nervous system function: behavioral skills involved in driving. J Stud Alcohol Suppl 10: 109-116.

Mongrain S, Standing L (1989). Impairment of cognition, risktaking, and self-perception by alcohol. Percept Mot Skills 69: 199-210.

Moskowitz H, Sharma S (1974). Effects of alcohol on peripheral vision as a function of attention. Hum Factors 16: 174-180.

National Highway Traffic Safety Administration (2002). DOT HS 809606.

Neill RA, Delahunty AM, Fenelon B (1991). Discrimination of motion in depth trajectory following acute alcohol ingestion. Biol Psychol 31: 1-22.

Ogawa S, Lee TM, Kay AR, Tank DW (1990). Brain magnetic resonance imaging with contrast dependent on blood oxygenation. Proc Natl Acad Sci 87: 9868-9872.

Peterson JB, Rothfleisch J, Zelazo PD, Pihl RO (1990). Acute alcohol intoxication and cognitive functioning. J Stud Alcohol 51: 114-122.

Ridderinkhof KR, de Vlugt Y, Bramlage A, Spaan M, Elton M, Snel J et al (2002). Alcohol consumption impairs detection of performance errors in mediofrontal cortex. Science 298: 2209-2211.

Rimm DC, Sininger RA, Faherty JD, Whitley MD, Perl MB (1982). A balanced placebo investigation of the effects of alcohol vs. alcohol expectancy on simulated driving behavior. Addict Behav 7: 27-32.

Schmithorst VJ, Holland SK (2004). Comparison of three methods for generating group statistical inferences from independent component analysis of functional magnetic resonance imaging data. J Magn Reson Imaging 19: 365-368.

Talairach J, Tournoux P (1988). A Co-Planar Sterotaxic Atlas of a Human Brain. Thieme: Stuttgart.

van de Moortele PF, Cerf B, Lobel E, Paradis AL, Faurion A, Le Bihan D (1997). Latencies in fMRI time-series: effect of slice acquisition order and perception. NMR Biomed 10: 230-236.

Verster JC, Volkerts ER, Verbaten MN (2002). Effects of alprazolam on driving ability, memory functioning and psychomotor performance: a randomized, placebo-controlled study. Neuropsychopharmacology 27: 260-269.

Voas RB, Tippetts AS (1999). DOT HS 808980.

Volkow ND, Mullani N, Gould L, Adler SS, Guynn RW, Overall JE et al (1988). Effects of acute alcohol intoxication on cerebral blood flow measured with PET. Psychiatry Res 24: 201-209.

Walter H, Vetter SC, Grothe J, Wunderlich AP, Hahn S, Spitzer M (2001). The neural correlates of driving. Neuroreport 12: 1763-1767.

Weiler JM, Bloomfield JR, Woodworth GG, Grant AR, Layton TA, Brown TL et al (2000). Effects of fexofenadine, diphenhydramine, and alcohol on driving performance. A randomized, placebo-controlled trial in the Iowa driving simulator. Ann Intern Med 132: 354-363.

Worsley KJ, Friston KJ (1995). Analysis of fMRI time-series revisited-again. Neuroimage 2: 173-181. 